\title{
Einseitige Berichterstattung
}

\author{
Alfred Mugglia, Georgios Dimitrakoudis ${ }^{b}$, Walter Grete ${ }^{c}$, Thomas Hardmeierd, Georg Ruckstuhle, \\ Ulrich Sigrist ${ }^{f}$, Alfred Stahelg, Willy Stoll' ${ }^{\mathrm{h}}$, Armin Wildi, Heidi Wolf Pagani
}

\begin{abstract}
${ }^{a}$ Dr. med., FMH Allgemeine Medizin, Steckborn TG, ehemaliger Präsident Thurgauer Ärztegesellschaft, Alt-Kantonsarzt Thurgau; ${ }^{b}$ Dr. med., FMH Anästhesiologie, Kreuzlingen, ehemaliger Leitender Arzt Anästhesie Kantonsspital Münsterlingen; ${ }^{c}$ Dr. med., FMH Allgemeine Medizin, Bachenbülach, ehemaliger Präsident der Zürcher Ärztegesellschaft; ${ }^{d}$ Prof. Dr. med., Winterthur, ehemaliger Chefarzt Pathologie Kantonsspital Münsterlingen; ${ }^{e}$ Dr. med., FMH Psychiatrie und Psychotherapie, Kreuzlingen, ehemaliger Leiter des Externen psychiatrischen Dienstes Münsterlingen; ${ }^{\dagger}$ Dr. med., FMH Allgemeine Innere Medizin, Weinfelden; ${ }^{9}$ Dr. med., FMH Allgemeine Medizin, Winterthur, ehemaliger Präsident der Paritätischen Kommission der Ärztegesellschaft Thurgau sowie Kantonsarzt-Stellvertreter Thurgau; ${ }^{h}$ Prof. Dr. med., Spezialarzt FMH Geburtshilfe/Gynäkologie, ehemaliger Chefarzt Frauenklinik Kantonsspital Aarau, ehemaliger Präsident der Spitalleitung des Kantonsspitals Aarau; i Dr. med., ehemaliger Leitender Arzt Pneumologie und Chefarztstellvertreter Medizinische Klinik Kantonsspital Münsterlingen; ' Dr. med., FMH Neurologie, Cureggia TI
\end{abstract}

Der Zeitgeist der 1950er Jahre lässt sich nicht mit den heutigen Wertvorstellungen, medizinischen Richtlinien und ethischen Grundsätzen gleichsetzen. Doch genau dieser Fehler wurde in den meisten Berichterstattungen rund um die Medikamentenforschung an der Psychiatrischen Klinik Münsterlingen gemacht. Damit entstand in der Öffentlichkeit ein einseitiges, negatives Bild über die Forschungstätigkeiten Roland Kuhns, welches die positiven Aspekte seines Wirkens völlig vergessen lässt.

Eine Kommission unter der Leitung der Historikerin Marietta Meier hat im Auftrag des Regierungsrates des Kantons Thurgau die Forschungsarbeit von Prof. Dr. med. Roland Kuhn anhand der umfangreichen Dokumentation untersucht und mit dem Buch Testfall Münsterlingen [1] eine interessante und umfangreiche sozialgeschichtliche Analyse über die Zeit zwischen 1940 und 1980 verfasst. Einzelne Medienberichte haben in der Folge mit reisserischen Überschriften die Forschungstätigkeit von Roland Kuhn kommentiert: "Versuche an Patienten machten Psychiater reich» [2], «Der fanatische Pillentester» [3], «Wie Oberarzt ‘Daddy Long Leg' seine Patienten als Versuchskaninchen benutzte» [4]. Wir waren über diese Schlagzeilen erschüttert und machten uns Gedanken, wie es zu einer derart negativen Berichterstattung kommen konnte.

\section{Fehlende ethische Grundsätze}

Die Kommission Meier hat es leider unterlassen zu betonen, dass die dazumal in Münsterlingen betriebene Forschung weit über den Kanton hinaus üblich war. Es ist bekannt, dass auch an anderen grossen Psychiatrischen Kliniken gleiche Medikamententests durchgeführt wurden. Es gab erst 1964 die «Deklaration von Helsinki» (Ethische Grundsätze für die medizinische Forschung am Menschen), und die Schweizerische
Akademie der Medizinischen Wissenschaften (SAMW) hat 1970 erstmals medizinisch-ethische Richtlinien für «Forschungsuntersuchungen am Menschen» verabschiedet. Der Kanton Thurgau hat als erster Kanton der Schweiz 1988 eine Ethikkommission gegründet, welche Forschungsprojekte beurteilte und genehmigte. Wir sind uns bewusst, dass die Art von Forschung von Roland Kuhn aus heutiger Sicht nicht mehr zulässig wäre, aber es wäre auch falsch, die damalige Forschungstätigkeit mit den heutigen Wertvorstellungen über die Forschung am Menschen zu beurteilen [5].

\section{Breite Unkenntnis der Behandlungs- möglichkeiten}

Walter Grete, Hausarzt und ehemaliger Präsident der Ärztegesellschaft Zürich, hat in der NZZ in einem Leserbrief die schwierige Situation der dazumal als «Irrenanstalten» bezeichneten Institutionen geschildert:

«Die Berichterstattung über die Erprobung neuer Substanzen in psychiatrischen Kliniken vor sechzig Jahren zeigt eine breite Unkenntnis der damaligen Behandlungsmöglichkeiten. Was wir heute Klinik nennen, war in den fünfziger Jahren bis weit in die sechziger Jahre hinein einfach eine Irrenanstalt, wo Wärterinnen und Wärter teilweise tobende und 
schreiende Wahnkranke 'bändigen' mussten. Daneben stierten kontaktlose, verstummte, schwer depressive Patienten reglos in den Tag hinein. Mauern grenzten die Anstalten gegenüber einer Gesellschaft $a b$, die überzeugt davon war, mit der Einrichtung einer Kost- und Logisstätte für Menschen, die ihre Eigenständigkeit verloren hatten, das menschlich Richtige getan zu haben. Aber das Gefühl von ärztlicher Hilflosigkeit dürfte alle, die dort arbeiteten, geprägt haben.

In dieser Situation war der Wunsch, mit medikamentösen Mitteln das Leid zu lindern und damit das Los der Betroffenen zu verbessern, allgegenwärtig. Wer ärztliche Verantwortung trug, der musste sich, getrieben von Mitleid und Menschlichkeit, um neue Therapieansätze kümmern. Die Kaderärzte in psychiatrischen Kliniken waren gefordert.»

\section{Unzureichende Infrastruktur und pre- käre finanzielle Situation}

In Münsterlingen wurden in den fünfziger Jahren bis zu 700 Patienten stationär betreut. Neben einer schlechten Bezahlung der Ärzte und Ärztinnen sowie des Personals verdiente auch die Infrastruktur keineswegs die Bezeichnung Klinik. Die Patienten wurden in grossen Sälen mit bis 20 Betten betreut, die sanitären Anlagen waren absolut unzureichend, und die Arbeitsfähigen mussten für Kost und Logis im Garten, in der Küche oder im benachbarten staatlichen Gutsbetrieb arbeiten. Im Buch Testfall Münsterlingen wurde der schwierigen finanziellen Lage des Kantons zu wenig Beachtung geschenkt. Die Einführung von Largactil ${ }^{\circledR}$ im Jahre 1954 hat in der Psychiatrie zu einer eigentlichen Revolution geführt. Unruhige und aggressive Patienten mussten nicht mehr in Zwangsjacken und Gummizellen behandelt werden, auch Insulinkuren, Deckelbäder oder sogar die Entfernung des Frontalhirns (Lobektomie) gehörten der Vergangenheit an. Das aus Frankreich stammende Largactil ${ }^{\circledR}$ war sehr teuer, so dass Kuhn kurz nach der Einführung dieses Medikamentes in der Schweiz beim Regierungsrat

\section{Im Buch Testfall Münsterlingen wurde der schwierigen finanziellen Lage des Kantons zu wenig Beachtung geschenkt.}

einen Nachtragskredit von 12000 Franken beantragen musste. Dem Kanton Thurgau kamen deshalb die Medikamententests von Kuhn nicht ungelegen, konnte doch der Staat dadurch die Kosten für Medikamente sparen. Wir sind auch der Meinung, dass der Staat bei der Aufsicht nicht versagt hat. Die Aufsichtskommis- sion musste nicht intervenieren, da Kuhns Medikamententests weder gegen ein Gesetz noch gegen eine Verordnung verstiessen.

\section{Weltweit erstes Medikament gegen Depressionen}

Die Bedeutung der Entdeckung von Kuhn, dass Imipramin der Firma Geigy eine antidepressive Wirkung zeigte, wird im Buch Testfall Münsterlingen zu wenig gewürdigt. Imipramin kam 1958 als Tofranil ${ }^{\circledR}$ in den Handel. Dass in Münsterlingen weltweit das erste Me-

Dass in Münsterlingen weltweit das erste Medikament gegen Depressionen entdeckt wurde, wird unserer Meinung nach zu wenig betont.

dikament gegen Depressionen entdeckt wurde, wird unserer Meinung nach zu wenig betont. Am 15. August 1997, 40 Jahre nach der Entdeckung von Tofranil ${ }^{\circledR}$, erschien in der Thurgauer Zeitung ein Artikel mit der Überschrift: «Münsterlingen; eine Sternstunde der Psychiatrie». Depressionen gehören zu den häufigsten Krankheiten, und diese seelische Krankheit ist sehr gefährlich, weil sie zu schweren sozialen und menschlichen Problemen, sogar zu einer Selbsttötung, führen kann. 2016 nahmen sich im Thurgau 41 Menschen und in der Schweiz 1016 das Leben (ohne Exit). Weltweit rechnet man, dass alle 40 Sekunden ein Mensch Suizid begeht, was die Bedeutung der Entdeckung von Kuhn unterstreicht.

\section{Freie, unbelegte Interpretation}

Die Kommission Meier hat nicht nur minutiös die verschiedenen Testsubstanzen analysiert, sie hat auch berechnet, dass Kuhn aus seiner Forschungstätigkeit extrapoliert auf das Jahr 2019 ca. 8 Mio. Franken verdient habe. Daraus haben Journalisten den Schluss gezogen, dass Kuhn dank den Medikamententests reich geworden sei. Im Untersuchungsbericht gibt es nur wenige Hinweise, wie das Geld verwendet wurde. In vielen Kliniken wurden wissenschaftliche Assistenten und Laborpersonal mit Geld für Studien mit neuen Wirkstoffen bezahlt. Unter anderem wurden in Münsterlingen Beiträge für die Anschaffung eines Elektroencephalographen und an eine Laborstelle von der Pharmaindustrie ausgerichtet. Das Wort Bereicherung ist eine freie, unbelegte Interpretation. Dividiert man die 8 Mio. Franken durch die 41 Jahre, während welcher Kuhn zuerst als Oberarzt und später als Chefarzt in Münsterlingen tätig war, ergibt das ein zusätzliches Einkommen von jährlich 200000 Franken. Chefärzte 
der Kantonsspitäler erzielen dank ihrer privatärztlichen Tätigkeit in der Regel ein vergleichbares Zusatzeinkommen.

\section{Detaillierte Betrachtung der Todesfälle}

Die 36 Todesfälle müssten auch etwas genauer analysiert werden. Die zehn Toten, bei denen ein Zusammenhang mit der Verabreichung von Prüfsubstanzen in Erwägung gezogen wurde, erstreckten sich über einen Zeitraum von 23 Jahren (1947-1970). Sämtliche verstorbenen Patienten wurden sehr lange - bis zu 34 Jahre - in Münsterlingen behandelt. Zitat aus Testfall Münsterlingen: «Erstens waren die Verstorbenen mehrheitlich alt (7 waren 75 und älter), zweitens war ihr gesundheitlicher Allgemeinzustand miserabel, sie waren alle sehr geschwächt und standen in manchen Fällen zweifellos kurz vor dem Tod.» Bei den übrigen 26 der 36 Todesfälle wurde von Kuhn zwar ein Zusammenhang mit den Prüfsubstanzen in Erwägung gezogen, aber auch die von ihm angeordneten Autopsien konnten keine Beweise liefern. Die Schlagzeile von 36 Todesfällen wurde in der Presse hochgespielt und ohne Beweise als medikamentöse Folgen gesehen.

\section{Unkenntnis der damaligen Verhältnisse}

Roland Kuhn hoffte bei seiner Forschungsarbeit ein Medikament zu finden, welches das Los von psychisch Erkrankten lindern könne, denn er vermutete als Ursa- che - in Analogie zur Depression - eine gestörte Chemie der Hirnzellen.

Zusammenfassend zeigen die journalistischen Interpretationen zum vorliegenden Bericht eine beängstigende Unkenntnis der damaligen Verhältnisse. Der Zeitgeist der fünfziger Jahre wird in den Kommentaren am Heute gemessen, und dessen Akteure werden mit unseren Richtlinien unzulässig, ja leichtfertig, verurteilt. Unsere Zeit darf auf 70 Jahre naturwissenschaft-

\section{Der Zeitgeist der fünfziger Jahre wird in den Kommentaren am Heute gemessen.}

lichen Fortschritt zurückblicken. Dieser Fortschritt hat unseren Zeitgeist geprägt. Erst er hat erlaubt, dass wir heute auch psychisch schwerkranke Menschen als eigenständige Individuen mit guten Heilungschancen wahrnehmen dürfen. Dies verdanken wir den suchenden Pionieren der Psychopharmakologie von damals. Sie haben die Mauern der dazumal als "Irrenanstalten» bezeichneten Institutionen geschleift.

Literatur

1 Meier M, König M, Tornay M. Testfall Münsterlingen, Chronos Verlag Zürich; 2019.

2 Meile S, Flammer L. Versuche an Patienten machten Psychiater reich. Thurgauer Zeitung, 24. September 2019.

3 Meile S. Der fanatische Pillentester. Thurgauer Zeitung, 24. September 2019.

4 Krummenacher J. Münsterlingen: Wie Oberarzt «Daddy Long Leg» seine Patienten als Versuchskaninchen benutzte. NZZ, 23.9.2019.

5 Tribelhorn M. Kriminalisierte Vergangenheit. NZZ, 2019. 